\title{
Linx
}

Revue des linguistes de l'université Paris X Nanterre

9 | 1997

Émile Benveniste. Vingt ans après

\section{Une théorie, un songe : les énonciations de Benveniste}

Patrick Dahlet

\section{OpenEdition}

Journals

Édition électronique

URL : http://journals.openedition.org/linx/1049

DOI : 10.4000/linx.1049

ISSN : 2118-9692

Éditeur

Presses universitaires de Paris Nanterre

Édition imprimée

Date de publication : 1 avril 1997

Pagination : 195-209

ISSN : 0246-8743

\section{Référence électronique}

Patrick Dahlet, « Une théorie, un songe : les énonciations de Benveniste », Linx [En ligne], 9 | 1997, mis en ligne le 06 juillet 2012, consulté le 02 mai 2019. URL : http://journals.openedition.org/linx/1049 ; DOI : 10.4000/linx.1049

Département de Sciences du langage, Université Paris Ouest 


\title{
Une théorie, un songe : les énonciations de Benveniste
}

\author{
Patrick Dahlet
}

\begin{abstract}
C
ontrairement à ce que suggère sa réception conventionnelle, la théorie de l'énonciation de Benveniste ne se superpose pas à son appareil coïncidence.

La première a l'apparence provocatrice d'un truisme : si la théorie ne se confond pas avec le signalement de cet appareil c'est tout simplement parce qu'il n'y a pas de théorie, Benveniste ne dénommant jamais comme telle l'ensemble des propositions qu'il formule et retient sur le sujet, à la différence de quelqu'un comme Bally qui inaugure Linguistique générale et linguistique française par l'intitulé Théorie générale de l'énonciation (1932, 1ère partie, 1ère section).

La deuxième tient au fait que l'appareil formel lui-même reprend et spécifie des élaborations conceptuelles dont l'enchaînement et la localisation ne sont précisément pas acquis - de la disjonction de l'emploi des formes et de celui de la langue, à l'identification intégrale de l'énonciation à un acte d'appropriation de la langue corrélé à un régime indiciel particulier et délimité-.

Il suffit d'écouter Benveniste pour s'en convaincre, puisqu'il assume explicitement cette dimension transitionnelle dans l'article qui présente l'"Appareil formel de l'énonciation", au début en relativisant son innovation quand il annonce qu'il s'agit d' "une autre manière de voir les mêmes choses", et à la fin en relativisant le dispositif qu'il vient de formuler par l'annonce que "Bien d'autres développements seraient à étudier dans le contexte de l'énonciation" et l'indication de leurs déterminations - "changements lexicaux", "phraséologie", démarcation de l'"énonciation parlée" et de l'"énonciation écrite" - (1970, II, 79 et 88). Si l'on reconnait avec C. Normand la coexistence de "textes d'arrêt" et de "textes d'ouverture" $(1986,195)$ dans la réflexion de Benveniste, force est de constater que ce clivage est aussi actif dans les limites même du texte emblématique que constitue l'appareil formel.
\end{abstract}




\section{Patrick Dahlet}

La troisième considération concerne l'omniprésence du questionnement énonciatif dans l'oeuvre de Benveniste. Tout se passe comme si l'absence d'acte d'institution théorique et l'auto-dépassement de l'appareil formel avaient en effet pour contrepoint une théorisation constante de la compréhension du langage sous l'éclairage de l'énonciation. Il n'y a pas d'un côté un ensemble de textes centrés sur l'énonciation, réunis sous les intitulés "L'homme dans la langue" et, dans une proportion moindre, "La communication", au sein de chacun des deux volumes de Problèmes de linguistique générale (PLG) et de l'autre des études de linguistique générale ou de grammaire comparée qui ne voudraient rien en savoir. Il y a de l'énonciation de part en part, mais à un degré et d'allure variables, non pas systématisée en un concept unaire, mais systématisant des cohérences multiples et partielles.

Bien loin d'invalider la démarche, comme le laisse entendre une certaine divulgation, ne serait-ce qu'en ne parlant pas de ses lieux d'(im)probabilité, c'est ce remodelage incessant qui la fonde en formulant et en lançant simultanément des corps de définitions sur des trajectoires de fuite. La multiplication de ces connexions n'est certes pas étrangère à la difficulté de combiner la confirmation de l'objet minimum saussurien et l'observation d'un objet en extension maximale. Mais elle témoigne surtout des hautes exigences d'un raisonnement attaché à absorber des champs de ruptures dans un même domaine de signification dont les éléments ne sont pas donnés.

C'est du moins le point de vue que je soutiendrai ici en tentant de dégager la diversification, sur fond de conflits et d'alliances, du parcours énonciatif de Benveniste, tel qu'il ressort de l'ensemble des textes regroupés dans Problèmes de linguistique générale (donnés ici avec leur première date de publication et leur place dans les $P L G$, notés I ou II suivant le tome), que la problématique énonciative en constitue ou non le thème central. Je saisirai cette discontinuité à travers la délimitation d'abord des variations sémantiques du moténonciation lui-même dans le temps de l'oeuvre, puis de la coexistence de programmes concurrents, de localisation et d'étendue variables, avant de mettre ses tensions en rapport avec des ambitions conceptuelles qui, dans un même mouvement, les rassemblent, les excèdent et les relancent dans l'espace d'une langue en mutation énonciative.

\section{Le mot énonciation}

Concernant la déclinaison du mot énonciation, je constaterai d'abord qu'il émerge dès 1950, mais que Benveniste l'utilisera toujours avec une relative parcimonie, y compris dans certains des textes consacrés à l'Homme dans la langue - aucune occurrence dans "L'antonyme et le pronom en français moderne" et dans "La forme et le sens dans le langage", une seulement dans "La philosophie analytique et le langage", deux dans "La nature des pronoms" -. La succession des attributions sémantiques du mot suffit pourtant en ellemême à mettre en évidence quatre ordres d'approches de l'énonciation. 
1.1. La première, qui n'est pas la moins originale, est relative à la première occurrence du terme, en 1950 dans "La phrase nominale" (1950, I, 151167), exactement vingt ans, faut-il le rappeler, avant la sortie de l'appareil formel, et dans un texte qui n'est pas inclus dans "L'homme dans la langue" mais dans les "Fonctions syntaxiques", co-naissance d'autant plus stimulante à noter que c'est Benveniste lui-même qui, comme le précise M.D. Moïnfar $(1992,21)$, a organisé ce volume des PLG. Le terme est livré au pluriel dans le contexte suivant : "la phrase nominale convient si bien à ces énonciations où elle tend d'ailleurs à se confiner, sentences ou proverbes" (1950, I, 165). Si l'on relie cet emploi aux deux autres du même texte, à savoir "l'énonciation ainsi formulée" et "deux modes d'énonciation" et à la référence explicite à des "textes de genre différent" (1950, I, 164 et 166), on perçoit qu'on a bien affaire à une acception de l'énonciation comme moule de structuration socio-cognitive des énoncés, très proche de l'assimilation bakhtinienne des "genres du discours" à des "formes /.../ prescriptives /.../ de l'énoncé" pour le locuteur (Bakhtine, 1979, trad. 1984, 287).

Du même coup, c'est d'emblée une matérialité contrainte, aussi indispensable que la langue, mais distincte de sa structure, que Benveniste convoque en fonction explicative. Contrairement aux trois approches qui suivent, il faut souligner que, bien que primitive, cette acception n'a pas fourni la base d'un programme énonciatif propre - et on peut faire l'hypothèse que c'est parce que son historicité l'a dérobée à l'insertion linguistique exigée par Benveniste - mais ressurgit épisodiquement sous la forme de ce que j'appellerai plus bas un site, autrement dit une référence conceptuelle susceptible de s'agglomérer à des programmes différents, comme c'est le cas, on le verra, avec la mention de la fidélité postulée de l'"auteur /.../ à son propos d'historien" dans "Les relations de temps dans le verbe français" (1959, I, 241).

1.2. La seconde apparition que j'ai relevée, si l'on tient pour une unité les trois données précédentes, date de 1954, dans un emploi également au pluriel : "énonciations enregistrables"(1954,I,16). La notion d'énonciation prend ici le sens d'une suite d'émissions sonores, sans référence situationnelle, mais déjà susceptible, il faut le noter, d'être le versant visible d'une formation invisible, la "manifestation contingente d'une infrastructure cachée" (1954, I, 16). L'emploi au pluriel restera pertinent. On le retrouve ainsi en 1968: "les signes /.../ entrent dans des combinaisons régies par un code et qui permettent un nombre d'énonciations qui dépasse tout calcul" (1968,II, 97). Il détermine une seconde approche de l'énonciation, en tant que quantité de phrases grammaticalement réalisables, relevant de descriptions phonétiques ou transformationnelles. C'est elle qui , reprise sous les concepts d'"énonciation phonique" et en partie de "sémantisation de la langue", compose les deux premiers aspects du procès d'énonciation que l'appareil formel évoque sans les élaborer. 


\section{Patrick Dahlet}

1.3. La troisième mention du terme date de 1956 dans le syntagme "processus même de l'énonciation linguistique" (1956, I, 252). Elle inaugure le programme indiciel puisque c'est à ce moment-là, sous le double effet d'un dépassement de la particularité sonore et d'un glissement du produit au processus, que l'énonciation se voit reconnaître ce rôle de "conversion du langage en discours" et de "procès d'appropriation par le locuteur", accompagné déjà de tout le paradigme déictique, que l'appareil formel reprendra quinze ans plus tard en y ajoutant la modalisation des "grandes fonctions syntaxiques" (1970, II, 84). Entre les deux, on notera l'introduction de l'expression "syntaxe d'énonciation" en 1963 (1963, I, 25) et l'objectif de "légitimer la catégorie de la modalité" en 1965 (1965, II, 187).

Je voudrais faire observer que ce mouvement de généralisation en termes de processus admet parfaitement, deux ans plus tard, une nouvelle fragmentation de la notion d'énonciation par retour sur le produit, double fragmentation en fait puisqu'elle établit d'un côté une distinction entre "énonciation "subjective"'", assertée par je, et "énonciation "non subjective", rattachée à la 3e personne, et de l'autre l'existence d'une forme singulière d'énonciation, de valeur performative, que Benveniste démarque explicitement par un démonstratif : "Cette énonciation est un accomplissement" (1958, I, 265). On remarquera que cette valeur performative, bien qu'elle fasse à elle seule l'objet d'un article ultérieur (1963, I, 276), ne fondera pas un programme. La raison en est, me semble-t-il, que Benveniste reporte d'emblée la force performative de l'évènement que constitue l'énoncé sur la "'subjectivité" du discours qui la rend possible" (1958, I, 265), c'est-à-dire sur la catégorie centrale du programme indiciel.

1.4. La dernière contextualisation de la notion d'énonciation que je pointerai est contenue dans l'invitation de Benveniste à "rechercher COMMENT s'effectue cette transposition d'une énonciation verbale en une représentation iconique", exprimée au bas d'une note de "Sémiologie de la langue" (1969, II, 59, n¹). En contraste avec la fragmentation précédente, la qualification d'énonciation verbale opère une surgénéralisation de la notion, quasi-clandestine puisque reléguée en note. Elle fonctionne ainsi comme désignation du système sémiologique de la langue dans son ensemble et principe de sa distinction face à d'autres systèmes. On pressent ici une volonté de remaniement considérable $\mathrm{du}$ patron linguistique saussurien: de la langue, principe d'unité et de définition de la sémiologie, on passe à l'énonciation, principe d'unification de la langue - le concept d'énonciation verbale fusionnant par avance la double signifiance, sémiotique et sémantique de la langue, introduite quelques pages plus loin (1969, II, 63) - et pour cette raison principe de classification et d'intégration inter-sémiologique.

Ce n'est sans doute pas un hasard que cet emploi surgénéralisateur figure dans le texte qui, juste après avoir insisté sur l'exigence de deux appareils conceptuels différents pour chacun des deux domaines de la langue, 
appelle à l"'élaboration d'une métasémantique qui se construira sur la sémantique de l'énonciation" et qui "sera une sémiologie de "deuxième génération"' (1969, II, 66), autrement dit un système en mesure d'intégrer dans une même représentation et de décrire de façon homogène ces deux ordres de langue qui viennent précisément d'être redéfinis comme séparés. Il y a là cette fois le motif, non pas d'un site, mais bien de tout un programme, que le choix ultérieur de l'appareil formel ne disqualifie pas, loin s'en faut, puisque l'identification en même temps que la mise entre parenthèses de l'énonciation comme "sémantisation de la langue" s'accompagnent alors en note d'un renvoi intradiscursif explicite à l'étude "Sémiologie de la langue" (1970, II, 81, n¹).

Un détour par les avatars du terme appareil confirme son aspiration irrédentiste. Car ils débordent largement l'emploi unique d'appareil formel de l'énonciation (1970, II, 79). On retrouve en effet le terme appliqué non seulement à chacune des disciplines de la linguistique, "appareil de la phonétique et de la morphologie" par exemple (1954, I, 289), mais également à chacune des deux dimensions complémentaires de l'énonciation dans le raisonnement de Benveniste : le discours, à un moment donné titulaire aussi d'un "appareil formel du discours" (1959, I, 239), et la langue, dont il est dit qu'elle "est en elle-même le lieu d'un travail incessant qui agit sur l'appareil formel, transforme ses catégories et produit des classes nouvelles" (1967, II, 160) ou qui est assimilée à l'"appareil de dénotation commun à tous" (1968, II, p.99).

$\mathrm{Au}$ fil de ces bifurcations, toujours signe d'une pensée à la recherche de l'univers qu'elle investit, on aura été sensible à l'autogermination dans la langue d'un homologue de l'appareil formel de l'énonciation.

\section{Les programmes et les sites}

Le foisonnement du mot énonciation dit assez l'amplitude de l'entreprise de Benveniste et suffit à bloquer sa réduction à un programme unique et normalisé. Mais les croisements de ses valeurs indiquent aussi des combinaisons autour d'une même problématique et des contributions à un horizon commun. C'est la diversité en même temps que la co-orientation de ces programmes énonciatifs, qui dessinent et partagent des frontières dans une nébuleuse, que je voudrais maintenant circonscrire.

Je le ferai en établissant une distinction entre des programmes, comme séquences d'actions finalisées, eux-mêmes effectifs ou virtuels, et des sites définis comme des éléments conceptuels isolés en mesure de s'articuler à chacun de ces programmes. Il ne s'agira bien sûr pas de décrire des contenus connus, mais de situer synthétiquement des différences et de clarifier des complémentarités dans un cheminement qui se construit.

Trois programmes peuvent rendre compte du développement de l'énonciation chez Benveniste, du moins c'est ce que je considèrerai ici : un programme indiciel, un programme perceptif, un programme métalinguistique. À ces démarches qu'on estimera effectives, s'adjoignent les deux programmes 


\section{Patrick Dahlet}

virtuels, de l'énonciation phonique et de la sémantisation de la langue, mentionnés en tant que tels dans l'"Appareil formel de l'énonciation" (1970, II, 80-81) et sur lesquels je ne reviendrai pas (cf. supra l'évolution du mot énonciation).

\subsection{Le programme indiciel}

Le programme indiciel est célèbre au point d'avoir longtemps prévalu comme référence unique du traitement de l'énonciation dans l'oeuvre de Benveniste.

Focalisant l'énonciation sous l'angle de l'acte qui la rend possible, sa pertinence ne repose pas seulement sur la description méthodique de ses paramètres et conditions d'organisation: "l'acte même, les situations où il se réalise, les instruments de l'accomplissement" (1970, II, p.81). Cette volonté méthodique est certes fondamentale, d'autant qu'elle transforme un effort ancien de Benveniste qui, mettant en question les "critères /.../ trop souvent "impressifs'"' utilisés pour analyser les manifestations linguistiques de 1"'attitude du parlant", en appelle dès 1954 à une "méthode applicable à ces contenus affectifs, à l'intention qui les suscite aussi bien qu'à la langue qui en fournit l'instrument" (1954, I, p.16).

Mais la caractéristique essentielle et inédite du programme est ailleurs. Elle est de considérer simultanément la "référence" comme "partie intégrante de l'énonciation" (1970, II, p.82) et le sujet comme élément intégré, en tant que locuteur, à cette référence: "je /.../ définissant l'individu par la construction linguistique particulière dont il se sert quand il s'énonce comme locuteur" (1956, I, p.255). Le sujet ne fait pas que communiquer, il est identifié par son acte en se représentant en train de l'accomplir.

Liée à la réévaluation, constitutive du programme indiciel, de la fonction monstratoire contre la fonction dénominatoire du langage, cette conception du sujet dans sa langue n'en est pas la conséquence automatique, loin de là, si l'on songe à cette longue tradition linguistique qui, tout en étant sensible aux conditions de l'énonciation, tend à la traiter comme une fonction ajoutée qui ne subvertit pas l'autonomie de la sémantique. De fait, si l'origine à trois aspects, le moi-ici-maintenant personnel, spatial et temporel, à partir de laquelle Benveniste organise sa perspective, n'est établie à la source de l'énoncé que pour autant qu'elle est formulée par lui, c'est précisément parce qu'il ne détache pas l'instance énonciative de la langue elle-même. En référence à l'hypothèse structurale, le sujet est visé très tôt dans l'immanence de la signification. Quand le pronom devient central, c'est l'affirmation qu'une "théorie linguistique de la personne verbale ne peut se constituer que sur la base des oppositions qui différencient les personnes" qui le devient aussi (1946, I, 227).

On perçoit ici un triple garde-fou contre le reflux du sujet vers le monde, en l'occurrence le redoublement de la médiation linguistique - théorie linguistique, personne verbale - associé à la convocation d'une analyse strictement interne. L'attention à cette médiation subvertit alors la propre fonction ostensive des 
indices ou des indicateurs de la personne. Contrairement à ce que leur sens fait imaginer, ils ne montrent plus rien, sinon un ensemble d'instructions qui confrontent les interlocuteurs à une mémoire énonciative : "On a traité trop légèrement et comme allant de soi la référence au "sujet parlant"/.../. C'est pourtant un fait à la fois original et fondamental que ces formes "pronominales" ne renvoient pas à la "réalité" ni à des positions "objectives" dans l'espace ou dans le temps, mais à l'énonciation chaque fois unique, qui les contient, et réfléchissent ainsi leur propre emploi" (1956, I, 254).

Au prix d'un réexamen de la logique de ses termes, qui deviennent des "signes "vides" non référentiels" (1956, I, 254) ou des "indicateurs autoréférentiels" (1956, I, 255), le programme indiciel se développe en fait comme auto-référentiel et aménage probablement par là son concept majeur, celui d'un sujet, "réalité du discours" (1958 I, 261), qui n'est plus identifié à aucune chose, mais à la contingence d'un acte à travers la nécessité d'une structure.

Dans ces conditions, l'absence du syntagme sujet de l'énonciation, relevée par C. Normand dans la mosaïque benvenistienne désignative du sujet, peut certes faire douter, comme elle le suggère, de la détermination de Benveniste à formuler une théorie du sujet (1986, 201-202), mais peut aussi se comprendre, je crois, comme le signe de son extrême vigilance à ne pas faire une théorie du sujet parlant qui soit celle d'un substrat qui pense. Car, pris au mot, l'expression sujet de l'énonciation renvoie à de l'énonciation qui $a$ un sujet connu avant son acte, ce qui est par définition contradictoire avec la représentation benvenistienne que le "locuteur se pose comme sujet, en renvoyant à lui-même comme je dans son discours" (1958, I, 260), autrement dit avec l'idée que le sujet ne peut fondamentalement être connu, dans la mesure où il peut l'être, que comme propriété de ce qu'il énonce.

\subsection{Le programme perceptif}

Le programme perceptif est littéralement complémentaire du précédent, puisqu'il est essentiellement à l'oeuvre dans les textes qui ne prennent pas explicitement pour enjeu la thématique énonciative. Pour fixer les contours de ce programme, longtemps ignoré, je donnerai d'emblée parmi d'autres possibles trois observations analytiques de Benveniste à des périodes différentes.

En 1949, il distingue les propositions latines pro et prae, en posant que la première signifie un "'en avant" /.../ relation objective, qui n'est pas exposée à s'inverser si la position de l'observateur change" alors que la deuxième indique la "position /.../"à l'avant" d'un objet" impliquant que le "sujet est censé constituer ou occuper la partie postérieure" (1949,I,133). En 1954, il établit que ce qui caractérise le mot sanscrit pánthah "est qu'il n'est pas simplement le chemin en tant qu'espace à parcourir d'un point à un autre/.../, il peut varier avec celui qui le parcourt/.../ C'est bien plutôt un "franchissement" tenté à travers une région inconnue et souvent hostile" (1954, I, 297). En 1960 enfin, étudiant le rapport de possession instauré par habeo pecuniam, il précise que "Le 


\section{Patrick Dahlet}

"ego" posé /.../ comme sujet n'est pas pour autant l'agent d'un procès : il est le siège d'un état, dans une construction syntaxique qui imite seulement l'énoncé d'un procès" (1960, I, 197).

Ce que font ressortir ces trois exemples, c'est une acception tout à fait particulière de l'énonciation au regard de celle du programme indiciel : moins les valeurs d'un sujet en acte que celles d'un objet pour le sujet.

Le sens de chacune des prépositions latines provient de ce qu'elles sont ou ne sont pas dans la continuité sensible du sujet. C'est sous l'éclairage de l'incertitude et du péril qu'il implique pour le sujet que le chemin prend la dimension d'un franchissement non acquis. Dans l'emploi indiqué avoir dénote le possesseur, repère de l'énoncé, comme fondamentalement affecté par l'objet auquel il consent. On saisit la portée pour les figures du sujet de l'intelligibilité discursive de ses objets. C'est l'interjection du sujet passionnel dans le sujet performant du programme indiciel. Cette tension pathique de l'énonciation est reconduite ultérieurement, avec peut-être encore plus de force, dans l'analyse sémantique de la préposition allemande vor. Considérant que vor marque "la position dans laquelle on se trouve sous l'impulsion d'une force irrésistible qui vous meut vers l'avant", Benveniste propose une interprétation unitaire des locutions vor dem winde segeln, "cingler devant le vent", et vor Freude weinen, "pleurer de joie" : le "comportement involontaire ("weinen"/.../) du sujet est assimilé au mouvement également involontaire du voilier ("segeln"), et le paroxysme de l'émotion impulsant à la force impulsante du vent" (1972, II, 140).

Au-delà de l'esthétique poignante de la comparaison, ou sous sa détermination (!), et du rapprochement qu'on serait tenté de faire avec la configuration d'une volonté indirectement active chez Saussure - "Il n'y a pas d'actes purement mécaniques, seulement des actes indirectement volontaires" (in Parret, 1993, p.207) - ce qui s'accentue ici c'est le rapport entre l'immanence $\mathrm{du}$ sensible et les dispositions du sujet, et du même coup la proximité avec l'option phénoménologique, argumentée par J. C. Coquet (1992). L'ordre du sensible et celui du sujet s'influent et se pondèrent, l'un l'autre. Mais ce que l'on pressent dans la montée du motif de l'impulsion, c'est que la position régissante serait d'abord ici celle de l'objet, d'où serait concédée une place au sujet.

\subsection{Le programme métalinguistique}

Le programme métalinguistique repose sur une conviction forte, "Il y a une métalangue" (1968, II, 97), et sur une exigence théorique, celle de "deux linguistiques différentes" relatives à "deux univers différents" (1962, I, 130), prise en charge par la distinction des deux domaines, sémiotique et sémantique, de la langue (1969, II, p.65). Et dans le cadre de cette distinction, il est clairement annoncé que c'est "Le domaine sémantique /.../ reconnu comme séparé" qui "aura besoin d'un appareil nouveau de concepts et de définitions"(1969, II, 65). De ce programme, je me contenterai de mettre en évidence trois caractéristiques. 
La première c'est qu'il émerge, et ce n'est pas un hasard, en conclusion de la proposition d'une représentation homogène des variations sémantiques des prépositions pro et prae: "La tâche entraîne l'obligation de réinterpréter toutes les données acquises et de refondre les catégories établies" (1949, I, 139). La deuxième, c'est qu'il est en permanence débordé par ces fondements, la mise au point d'un "corps de définitions rigoureuses" pour les "notions sémantiques" impliquant "à leur tour une discussion sur les principes mêmes de la signification" (1954, I, 289). La troisième est que la possibilité d'une métalangue, adéquate au domaine sémantique et donc aux phénomènes énonciatifs en particulier, est donnée comme une propriété même de la dimension énonciative, puisqu'il s'agit de "créer un deuxième niveau d'énonciation, où il devient possible de tenir des propos signifiants sur la signifiance" (1969, II, 65).

Toute description se double ainsi pour Benveniste d'un devoir de renouvellement métalinguistique en mesure d'incorporer la fonction discriminatoire de la subjectivité, devoir harassant car obligé de produire simultanément des catégories métalinguistiques, comme discours sur des propriétés sémantiques et énonciatives, et des analyses empiriques de leurs modes de signifiance et de leurs lieux de détermination.

\subsection{Les sites discursifs, cognitifs et symboliques}

À la croisée de ces programmes, le texte de Benveniste révèle aussi des sites énonciatifs, unités conceptuelles isolées susceptibles d'être mis en correspondance avec eux ou d'en cristalliser d'autres cohérences. Il me semble à cet égard pouvoir discerner trois sites que je localiserai sans trop m'arrêter sur leur organisation.

2.4.1. Le premier renvoie à l'inscription, déjà évoquée, de l'énonciation dans le cadre du genre. On en trouve l'illustration dans des analyses syntaxiques, comme celle de la phrase nominale, qui peuvent être "fondées sur des textes de genre différent" (1950, I, 164), ou énonciatives, comme celle du système des temps en français, qui met en relation les marques du récit historique avec les régularités du "propos d'historien", et celles du discours avec les "correspondances, mémoires, théâtre, ouvrages didactiques, bref tous les genres où quelqu'un s'adresse à quelqu'un" (1959, I, 241-242). On notera que la référence au genre apparaît aussi dans les travaux comparatistes. C'est ainsi que Benveniste justifie la "remarquable constance d'emploi" d'exemples perses de parfait par le "caractère formulaire du texte" observé $(1952,54)$.

2.4.2. Le second site, concomitant du précédent dans les écrits de Benveniste, introduit les processus cognitifs, au sein du récit historique toujours, qui est supposé proscrire "discours, réflexions, comparaisons" (1959, I, 241) et être par définition une actualisation de raconter, mais aussi dans la structuration de formes syntaxiques, les verbes "comprendre, découvrir, remarquer, voir" 


\section{Patrick Dahlet}

étant par exemple envisagés comme des réalisations privilégiées de habere + participe passé au parfait parce que dénotant un "procès "sensori-intellectuel" intérieur au sujet" (1966, II, 129), ou encore à travers l'établissement d'une distinction entre des verbes modaux dits d'attitude, du type "je suppose, je présume", des verbes descripteurs d'opérations de pensée, tels que "je raisonne, je réfléchis, je pose, je résume " et des verbes dénotatifs d'un acte indidividuel de portée sociale, à l'instar de "jurer, promettre, garantir, certifier" (1958, I, 265).

2.4.3. Le dernier site fait intervenir l'énonciation en tant que condition de l'interprétation symbolique. Il se rattache bien sûr au thème des figures recensées dans "Remarques sur la fonction du langage dans la découverte freudienne": euphémisme, allusion, antiphrase, prétérition, litote, métaphore, métonymie, synecdoque, ellipse (1956, I, 87). Mais c'est un motif qui insiste. On le perçoit déjà, me semble-t-il, dans le "symbolisme" auquel achemine, suivant Benveniste, les études sur l'"ordre des mots, sur la qualité des sons, sur les rythmes et la prosodie comme sur les ressources lexicales et grammaticales de la langue" (1954, I, 16). Et on le retrouve encore dans l'approche de la blasphémie, considérée comme "domaine de l'expression émotionnelle /.../, qui a ses règles, sa syntaxe, son élocution" et identifiée à une "décharge émotive" qui "trouve issue dans une jaculation brutale" (1966, II, 257).

Le sujet qui blasphème partage donc la même façon de dire, sur le mode de la jaculation, que celui de la cure de parole psychanalytique. Avec l'évocation de Freud, cette coïncidence inciterait à faire du site symbolique un observatoire de l'inconscient à travers l'énonciation. Je serais pourtant plutôt porté à le rapprocher du programme perceptif. Comme le sujet qui pleure de joie, celui qui blasphème apparaît sous le coup d'une émotion irrésistible qui transforme l'acte d'énonciation du sujet, sans qu'il le veuille peut-être, mais pas nécessairement à son insu.

Ni prédit, ni inouï, le moi de l'énonciateur de Benveniste ne serait alors pas séparable de ce "MOI-sommeil" que Saussure, décrivant la pensée hindoue, tient pour le "parfait moi /.../ puisque nous avons là l'image du moi susceptible d'impressions mais n'en recevant aucune, en même temps sans conscience de son propre moi" (in Parret, 1993, 221-222).

\section{Des trajectoires qui comblent la langue}

Loin de conduire des développements théoriques hétérogènes ou d'exprimer des renoncements, voire des dissolutions internes, ces programmes et ces sites énonciatifs partagent une même question, la "question centrale de la signification" suivant les propres termes de Benveniste (1952, I, 117). De fait la reconnaissance de la signification organise de bout en bout l'ensemble de la démarche benvenistienne, condition d'organisation des données, pivot du raisonnement et horizon de ses représentations, comme le démontre C. Normand (1989 et 1992).

S'il est vrai que cette reconnaissance est constante, sa formulation 
pourtant se déplace. Dans les commencements ce qui est affirmé c'est que la "langue est informée de signification" (1954, I, 12). Mais ultérieurement ce qui sera légitimé c'est qu'au "fondement de tout il y a le pouvoir signifiant de la langue, qui passe bien avant celui de dire quelque chose" (1966, II, 229).

D'une formule à l'autre, l'emprise de la signification s'est considérablement étendue, au point de caractériser une nouvelle souveraineté. De présupposé de la description linguistique, la signification devient l'incarnation de la langue, principe d'allégeance à la langue de toutes les catégories du réel, y compris de sa propre fonction communicative. Benveniste impose alors une langue qui, loin d'être structurée par ce dont elle parle, construit ses objets dans l'ordre qui est le sien : "la langue /.../ crée donc des formes, des schèmes de formation ; elle crée des objets linguistiques qui sont introduits dans le circuit de la communication" (1968, II, 101). Et on aura noté qu'il attribue directement à la langue, et non pas au discours, cette activité de construction et d'aménagement d'objets. Le dépassement des vieux postulats rationalistes du langagecorrespondance est ici on ne peut plus manifeste (Dahlet, 1995, 159-161).

Cette consécration repose en profondeur, me semble-t-il, sur une densification énonciative de la langue, que je considèrerai ici comme le résultat de la rationalité croisée des programmes indiciels et perceptifs, elle-même tenue sous le charme d'un mot, celui récurrent du verbe énoncer.

\subsection{La langue en énonciation}

Si chacun de leur côté, les programmes indiciels et perceptifs développent une dimension de l'énonciation, leur conjonction amplifie notablement l'architecture énonciative de la langue, au point de la placer au fondement d'une langue qui serait réconciliée avec l'action de ses signes. Les deux programmes sont dans cette optique parfaitement complémentaires, dans la mesure où ils interposent tous les deux dans la langue le cours signifiant du langage, mais à des niveaux d'ouverture différents.

3.1.1. A cet égard, et au-delà même de la réévaluation significative de la deixis, la contribution particulière de la proposition indicielle passe bien entendu par l'affirmation du caractère foncièrement dialogique de l'acte énonciatif : "l'accentuation de la relation discursive au partenaire, que celui-ci soit réel ou imaginé, individuel ou collectif /.../ ce qu'on peut appeler le cadre figuratif de l'énonciation /.../ est donné nécessairement avec la définition de l'énonciation" (1970, II, 85). En situant l'énonciation dans la circulation du dire des autres, Benveniste revient sur l'évidence de l'inscription individuelle du sujet dans la langue : cette inscription n'est pas indépendante des variations du signifiant d'en face.

Mais ce qui fait toute l'originalité de ce dynamisme dialogique, c'est que la force de décentrement qu'il représente n'est pas séparable, dans le dispositif benvenistien, de l'auto-centrage - rappelons-le, "chaque instance de discours 


\section{Patrick Dahlet}

constitue un centre de référence interne" (1970, II, 82) - impliqué par la suiréflexivité établie au coeur de l'acte énonciatif. Ce qui ressort en effet d'une telle combinaison de décentrement et d'autoréférence, c'est que les signifiants d'en face sont nécessairement réentendus dans la forme linguistique qui "énonce la présente instance de discours" (1956, I., 252). J'oserai dire que les implications de cette réentente dans la réalité linguistique à laquelle s'identifie le je qui énonce, sont à la lettre substantielles : le je peut toujours se redire en substance ce qu'il a perçu de l'autre, ce qui du même coup conditionne l'organisation de cette substance à la production d'autres signifiants. Sur la scène d'un acte énonciatif finalisé par sa propre réalisation c'est ainsi, à chaque instant, la possibilité d'une (ré)articulation de la substance qui se joue.

3.1.2. Quant à la contribution essentielle du programme perceptif à l'incarnation énonciative de la langue, elle est, je crois, de distendre littéralement le champ de l'énonciation en rendant compte du fait que cet effet d'enoncé, auquel est par ailleurs identifiée l'instance d'énonciation, n'est pas forcément présent dans l'énoncé. L'énonciation n'est pas empiriquement contenue dans le recensement de ses marques traditionnelles. Celles-ci ne correspondraient qu'à une infime partie, la partie émergée, de la masse flottante.

Car toute prédication suppose pour Benveniste, en l'absence même d'une quelconque trace de prise en charge subjective, l'effet d'un je dis qui se porte garant de la réalité de l'objet qu'elle agence. Parmi d'autres, c'est bien ce qu'établit sa définition de la fonction assertive, concomitante à la première apparition, déjà notée, du terme énonciation: "A la relation grammaticale qui unit les membres de l'énoncé s'ajoute implicitement un "cela est!"qui relie l'agencement linguistique au système de la réalité" (1950, I, 154).

Le locuteur et l'énonciation peuvent ainsi n'être pas seulement posés ou énoncés, mais bien transposés. On a affaire à un développement elliptique de l'énonciation, qui oriente en fait régulièrement les analyses comparatistes et syntaxiques de Benveniste, comme on l'a vu en exemplifiant les options du programme perceptif. En tant que tel, parce qu'il est transposé dans le flux de la langue, le sens de cet acte énonciatif doit être reconstruit par un effort d'interprétation pour être lisible. Cette catalyse interprétative d'une structure absente peut être explicitement revendiquée par Benveniste. C'est notamment le cas dans la description des composés nominaux dits possessifs du type rougegorge, dont il est dit qu'ils incluent "à titre de facteur indispensable de la construction, l'attributaire actuel ou virtuel du "être-à" (1967, II, 156).

La conception d'une saillance implicite de l'énonciation est aussi envisagée, il faut le souligner, dans les textes de "L'homme dans la langue" où l'on peut relever, à propos des instances d'emploi de je et $t u$ qu'il "importe peu que ces formes doivent figurer explicitement dans le discours ou puissent y demeurer implicites" (1956, I, 253), ce qui confirme, si besoin est, la thèse de la complémentarité des programmes indiciels et perceptifs dans le sens d'une globalisation discursive de la langue. 


\subsection{L'énonciation sous le charme de son verbe}

$\mathrm{Au}$ croisement de ses programmes, l'énonciation tend ainsi à être virtuellement localisée partout où il y a signification. Dans ces conditions, l'énonciation ne doit plus seulement concourir, avec d'autres principes, à une saisie exhaustive de la signification, sachant qu'"on n'a jamais trop de toutes les déterminations pour définir un signe" (1966, II, p.239), mais elle devient potentiellement le medium unique de la totalité de la langue et de la signification pour le linguiste, ce qui ne va pas sans une part d'enchantement, que je voudrais situer pour terminer.

3.2.1. Cet enchantement, je le relie fondamentalement à l'investissement subjectif dont semble être l'objet dans le texte de Benveniste le représentant linguistique même du procès qu'il étudie, en l'occurrence le verbe énoncer .

Outre une fréquence d'emploi supérieure à la moyenne et qui s'accroît considérablement dans la dernière période, j'ai cru en effet remarquer que chacune des manifestations du mot est chargée de valeur incantatoire, ou pour le moins introductrice d'une communication cruciale pour la théorisation, comme en témoignent les quelques exemples suivants: "Enonçons donc ce principe : tout ce qui relève du sémiotique a pour critère nécessaire et suffisant qu'on puisse l'identifier au sein et dans l'usage de la langue" (1966, II, 232-233) ; "cette éclosion d'une linguistique théorique très exigeante, /.../ essayant /.../ de s'énoncer en structure organique" $(1968$, II, 16) ; "quand il a énoncé cela /.../ c'était une nouveauté importante" (1968, II, 32, à propos de Saussure) ; "Il est temps d'énoncer les conditions minimales d'une comparaison entre systèmes d'ordres différents" (1969, II, 56).

L'occurrence en discours du verbe énoncer renvoie ainsi chez Benveniste à un acte d'institution, constitutif et garant d'une réalité majeure, qui n'existait pas encore avant qu'il la formule et qui s'enlève dans l'effort de sa formulation, contre toute attente peut-être, mais différenciée sans aucun doute par la seule indication de ce mot au charme particulier, le verbe énoncer, qui suffit à la construire.

3.2.2. Parallèlement, et sans même évoquer l'enchantement de Benveniste devant la relance indéfinie du sens en discours, "cette tête de Méduse /.../ toujours là, au centre de la langue, fascinant ceux qui la contemplent" (1962, I, 126), c'est le fonctionnement en soi de l'énonciation, qui peut s'imposer à Benveniste avec les propriétés d'un sortilège, comme dans le cas de cette émouvante explication qu'il donne, de la formation du temps linguistique : "quelque chose de singulier, de très simple et d'infiniment important se produit qui accomplit ce qui semblait logiquement impossible : la temporalité qui est mienne quand elle ordonne mon discours est d'emblée acceptée comme sienne par mon interlocuteur. Mon "aujourd'hui" se convertit en son "aujourd'hui" /.../ quand il parlera /.../ je convertirai /.../ sa temporalité en la mienne" $(1965$, II, 76). 


\section{Patrick Dahlet}

Si l'énonciation en même temps qu'un domaine théorique est le champ d'un rêve, l'un des motifs du charme est ici on ne peut plus clairement explicité : l'énonciation réalise ce qui "semblait logiquement impossible". Elle est ce fil d'Ariane qui met sur la piste du nombre d'or, cette composition qui surprend toute expectative logique, celle que déclenche la représentation d'un langage organisé, au point, notons-le, d'aspirer dans sa démonstration un je"je convertirai"-, qui est aussi celui du linguiste ébloui par sa propre constitution énonciative.

Par un retour du rêve dans la théorie, la logique subjuguée est également une logique à repenser, parce qu'elle qualifie le fonctionnement ordinaire du langage, sa capacité à conquérir des identités en dépassant le projet qui le fonde. A la charnière de la langue et du discours émerge en effet un monde, qui à lui tout seul fait la signification parce qu'il englobe les deux autres dans une unique réalité : le "monde de l'énonciation" (1969, II, 64), dont l'empire unique cautionne par avance la réconciliation, dans la nouvelle totalité qu'il forme, des "deux linguistiques différentes" conçues par Benveniste pour penser l'une le sémiotique, l'autre le sémantique (1962, I, 130 et 1966, II, 236). Il existe bel et bien chez Benveniste, sous le signe de l'énonciation, une voix signifiante qui n'est pas de traverse. La preuve en est qu'il n'y a pas d'autre temps que le sien : "On pourrait croire que la temporalité est un cadre inné de la pensée. Elle est produite en réalité dans et par l'énonciation" (1970, II, 83).

Dans ces conditions, il pourrait bien se faire que cette logique à repenser soit celle de la représentation de la langue elle-même. Au détour d'une démonstration, Benveniste se prend à le dire : la "langue énonce" (1966, II, 226). Sur fond d'introjection énonciative et de projection de mondes, la langue s'anime, au sens où la théorisation de la langue s'absorbe en une théorie de langue, procession de discours en langue autant que reconstruction d'un système à l'image de son énonciation.

Mais avec l'apparition d'une "langue-discours" (1966, II, 229), il n'y a plus d'extérieur à la langue : elle est, sous l'effet de sa transmutation énonciative, sa seule réalité. Alors la question de son appropriation ne se pose plus, ou du moins plus dans les mêmes termes, ce qui contribuerait à expliquer que les analyses et la métalangue énonciatives de Benveniste soient lancées sur une trajectoire de fuite en même temps que l'absence de revendication explicite d'une théorie de l'énonciation, absence qui n'oblitère en rien le rôle matriciel du concept, bien au contraire. Car en rendant observable une autre langue, languediscours ou langue qui énonce, la rationalité énonciative constitue autant pour Benveniste un argument théorique qu'un irrremplaçable contexte heuristique : "découvrir les cadres dans lesquels on peut inventer" (1968, II, 28). 
Une théorie, un songe ; les énonciations de Benveniste

\section{Bibliographie}

BAKHTINE, M, (1979), Esthétique de la création verbale, Paris, Gallimard, 1984.

BALLY, C, (1932), Linguistique générale et linguistique française", Berne, A.Francke, 1964.

BALLY, C, (1939), "Qu'est-ce qu'un signe?", Journal de psychologie normale et pathologique, XXXVI, pp.161-174.

BALLY, C. (1940), "L'arbitraire du signe", Le Français moderne, VIII, n³, pp.193-206.

BENVENISTE, E, (1952), "La construction passive du parfait transitif", BSL, XLVIII, fasc. 1, pp.52-62.

BenVEniste, E, (1966), Problèmes de linguistique générale, Tome I, Paris, Gallimard.

BENVENISTE, E, (1974), Problèmes de linguistique générale, Tome II, Paris, Gallimard.

CoQueT, J.-C, (1992), "Note sur Benveniste et la phénoménologie", LINX, 26, pp.41-48.

DAHLET, P, (1995), "Les formes du sens dans l'interprétation", Lingua e literatura, 21, Université de São Paulo, pp. 145-168.

MOÏNFAR, M.D, (1992), "L'oeuvre d'Emile Benveniste", LINX, 26, pp.15-26.

MONTAUT, A, (1992), "La méthode de Benveniste dans ses travaux comparatistes : son discours et son sujet", LINX, 26, pp. 109-135.

Normand, C, (1986), "Les termes de l'énonciation chez Benveniste", Histoire, Epistémologie, Langage, tome 8 , fasc. II, pp. 191-206.

Normand, C, (1989), "Constitution de la sémiologie chez Benveniste", Histoire, Epistémologie, Langage, tome 11, fasc. II, pp.141-168.

NORMAND, C, (1992), "Benveniste : linguistique saussurienne et signification", LINX, 26, pp.49-75.

PARRET, H, (1993), "Les manuscrits saussuriens de Harvard", Cahiers Ferdinand de Saussure, 47, pp.179-234. 